\title{
In Situ Imaging Studies of Combustor Pressure Effects on Soot Oxidation
}

Anton D. Sediako $\square^{a}$, Anthony Bennett $\square^{b}$, William L. Roberts ${ }^{b}$, Murray J. Thomson *a $\square$ authors contributed equally to the work.

a University of Toronto, Department of Mechanical and Industrial Engineering 5 King's College Road, Toronto, ON M5S 3G8, Canada

b King Abdullah University of Science and Technology, Thuwal 23955-6900, Kingdom of Saudi Arabia, Al-Kindi (building 5), Level 4, Sea side, Left side

\section{Corresponding Author}

* Murray J. Thomson_ murray.thomson@utoronto.ca 
ABSTRACT: Airborne soot is a product of incomplete combustion from engines and industrial processes. Unburnt soot is carcinogenic, a major contributor to climate change, and detrimental to combustor lifespan and efficiency. An understanding of how high-pressure combustion affects the oxidation properties of soot is crucial for the design of clean-burning, high-pressure engines and downstream soot filtration technologies. This paper presents the first real-time look at the oxidation of soot particles formed at high-pressure and demonstrates that the oxidation pathway changes as combustor pressures increase. Soot particles were formed in an ethylene-fueled diffusion flame, with pressures ranging from 1 to 25 bar, and were subsequently sampled and oxidized inside an ETEM allowing for the nanoscale, real-time observation of oxidation pathways. The high-pressure generated soot grew larger in diameter, formed larger aggregates, and developed graphitic outer shells, protecting the reactive amorphous carbon core. The graphitic shell structure fundamentally changed the oxidation pathway of soot from diffusion driven internal oxidation at lower pressures to highly non-reactive surface oxidation reactions at high pressures. This work demonstrates that as combustor pressures increase to achieve higher thermodynamic efficiencies, highly resilient oxidation-resistant soot is produced.

\section{TOC GRAPHICS}

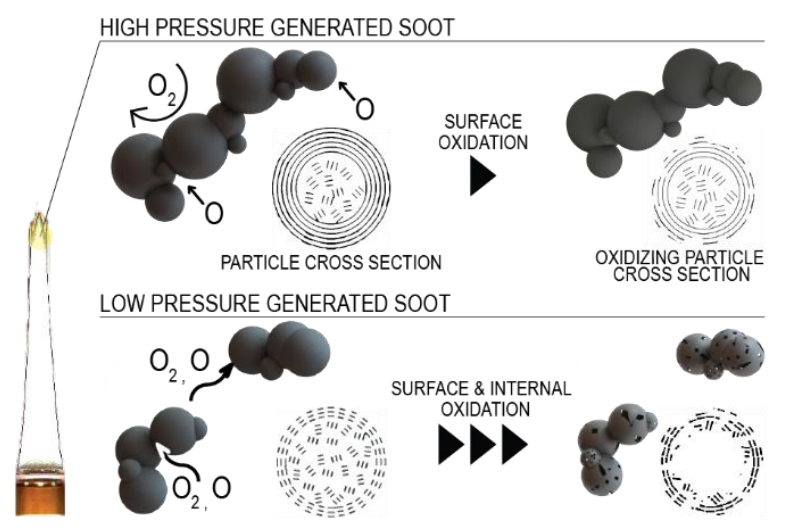




\section{INTRODUCTION:}

Despite increasing growth and use of renewable energies worldwide, the world's dependence on hydrocarbon fuels remains at an all-time high of $87 \%$ of total energy usage as of $2016^{1}$. Incomplete combustion of these hydrocarbon fuels releases solid carbon nanoparticles, i.e. soot or black carbon, into the atmosphere. Soot particles, penetrating deep into lung tissue and blood streams, correlate directly to long-term health problems such as cancer, asthma, and heart disease and are currently considered the most harmful airborne pollutant ${ }^{2}$. Apart from health effects, soot released from combustors is also the strongest sunlight-absorbing atmospheric pollutant and is currently the second highest contributor to climate change.

In order to increase thermodynamic efficiencies of engines, engineers have continuously driven up engine temperatures and operating pressures-with current diesel engines operating at pressures in excess of 100 bar. This increase in pressure changes the flame structure, reaction rates, and the type of soot produced. As the soot shape and structure changes, so do its health effects, its resistance to oxidation, and the filtration techniques needed to remove it. While previous studies have observed various modes of soot oxidation ${ }^{3,4}$, current soot models only assume surface reaction pathways. Using an in situ environmental electron microscopy technique (ETEM), our work aims to visualize and understand the effect of combustor pressure on the internal nanostructure of soot and the resulting oxidation pathways of these particles - establishing the need more complex soot oxidation models with internal nanostructure dependence.

\section{BACKGROUND:}

Soot formation begins with the pyrolytic breakdown of the fuel into unsaturated hydrocarbons (particularly acetylene), its heavier analogues, and polycyclic aromatic hydrocarbons ${ }^{5}$. These species serve as soot precursors, and, as temperatures grow, these building blocks combine from 
the gas phase into the first soot nuclei particles. As these soot nuclei move further through the flame, they grow, restructure, collide, and combine to form large soot aggregates. Studies have also found that, as the particles remain in high temperature regions of the flame, they begin to restructure from disordered carbon to structured graphitic carbon ${ }^{5-7}$. This transition is most clearly seen as a layered carbon "shell" around the soot particles. The general soot formation mechanism remains consistent for all hydrocarbon fuels, but environmental conditions and fuel chemistry can dramatically alter soot structure, aggregate size, and oxidation rates ${ }^{8}$. Changes in soot nanostructure has been shown to directly affect the rate of oxidation of soot ${ }^{4}$.

To date there have been numerous high-pressure experimental studies relying on laser, optical, and electron microscopy techniques. Flower and Bowman found in an ethylene flame that between 1 and 10 bar soot volume fraction increases exponentially with pressure ${ }^{9}$. This increase in soot volume fraction with pressure has been explained by Roberts et. al. ${ }^{10}$ : with increasing pressures and densities, reaction rates increase, the parent fuels are consumed earlier in the flame, and soot nucleating species are formed earlier and in much larger concentrations. Studies have also found that, apart from increased soot yield, increases in pressure lead to decreased soot oxidation rates ${ }^{9,11}$. It is hypothesized that these dramatic changes in oxidation rates are the result of a temperature drop in the flame caused by greater radiative heat losses from the higher quantities of soot.

These early studies confirmed the quantity of soot emitted from a flame is a result of the competition between soot formation and oxidation, yet they have made no connection between the nanostructure changes as a result of the pressure increase and the soot reactivity. Furthermore, a recent study using Raman spectroscopy found that only sampling height (hence residence time) and not pressure affected the nanostructure of soot sampled from a methane flame between 10 and 20 bar $^{12}$. Recent modeling mechanisms proposed by Appel et. al. ${ }^{13}$ also neglect potential complex 
oxidation pathways, assuming all soot oxidation occurs through surface oxidation. In this study, we show that the effects of pressure on the internal structure of soot cannot be neglected, as formation of soot earlier in the flame results in longer residence times - promoting not only soot growth, but also a transition from amorphous to graphitic soot structures as the particles are "annealed" in the flame.

As soot spends more time in high-temperature flame regions, its nanostructure rearranges into a graphitic shell around the soot particulates ${ }^{14}$. The primary particle cross section in Figure 1 shows an example of this. The shell is composed of dehydrogenated graphitic planes. Graphitic planes that are more ordered and have longer fringe lengths lack active sites for oxygen to attach to and result in particles that are more resistant to oxidation ${ }^{15}$. Holes or irregularities in the graphitic shell allow oxygen to diffuse into the particle resulting in soot oxidation. It can be expected then that longer residence times produce more graphitic particles, which oxidize more slowly and exclusively through surface reactions with high-temperature radical species such as $\mathrm{O}$ and $\mathrm{OH}$ 16,17. These radical species are present in both premixed engine flames as well as within the ionized environment inside of the ETEM ${ }^{18}$. While previous work studying soot formed in high-pressure conditions has established important rates and hypotheses, it has been limited to bulk experimental techniques or "snapshots" of the process (e.g. TGA, TEM) ${ }^{19}$. The downside of such techniques is that, due to the inability to observe and track individual particles as they oxidize, they are unable to definitively answer or confirm pathways on soot restructuring, oxidation modes, and oxidation pathways.

Our experimental method aimed to synthesize, collect, and oxidize soot from different combustor pressures to establish the internal nanostructure and oxidation pathways. The samples were synthesized in a high-pressure combustor and collected by thermophoretic deposition, and then 
they were oxidized and imaged inside the ETEM. We employed a variable pressure, nonpremixed, laminar co-flow flame setup (Figure 1 -A) fueled by nitrogen-diluted ethylene. The ethylene/nitrogen mixture provided a stable and representative soot source while maintaining a constant carbon flow rate ensured that any observed changes were strictly the result of changing pressures. Substrates were pneumatically injected through the flame at high speed (Figure 1-E), withdrawn, and then inserted into the microscope. Soot oxidation was then directly observed using the HF-3300 Environmental Transmission Electron Microscope (ETEM). Unlike conventional TEMs, which operate with the sample under high vacuum, making real-time oxidation impossible, the HF-3300 allows experimenters to surround the sample with a quiescent cloud of $\mathrm{O}_{2}$ (up to 1 $\mathrm{Pa}$-abs) and temperatures up to $1300 \mathrm{~K}$. The samples were first analyzed with ex situ highresolution electron microscopy to establish their internal nanostructure and then with in-situ electron microscopy to study how oxidation pathways change for soot collected at varying pressures.

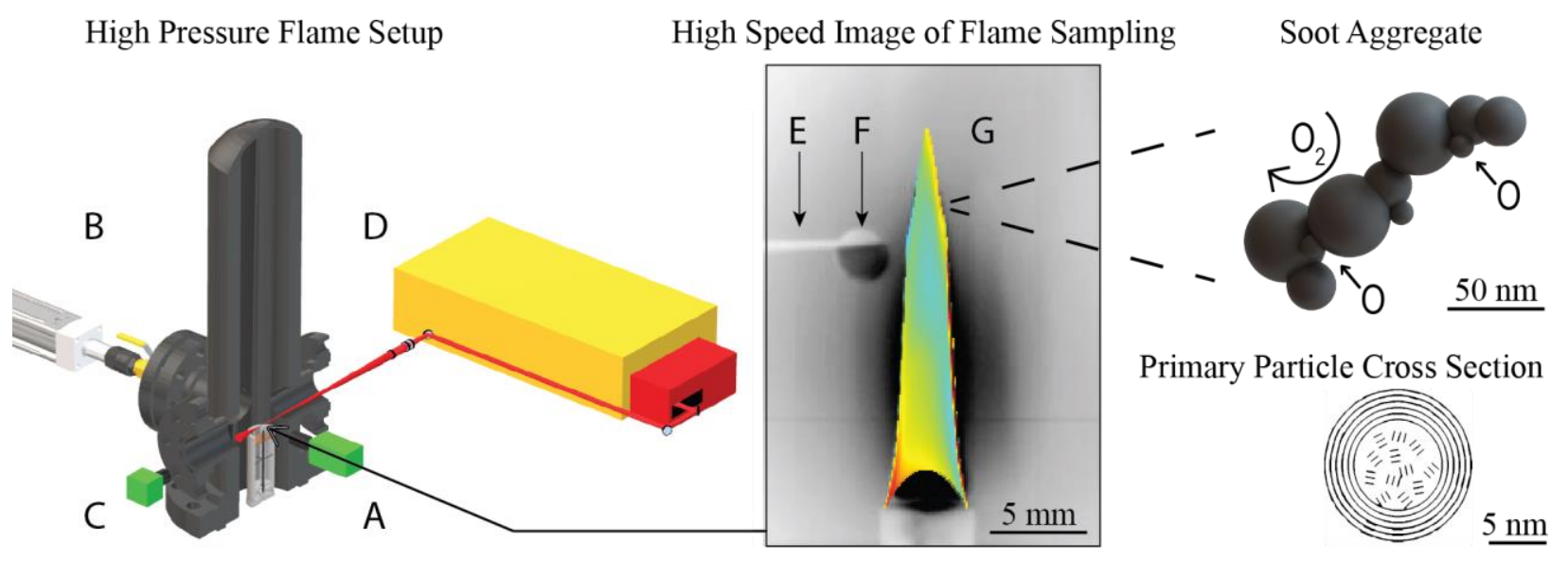

Figure 1: A: High Pressure Flame Vessel; B: Pneumatic Thermophoretic Sampling Injection Setup; C: High Speed Camera Setup; D: Laser Ignition System; E: Thermophoretic Sampling Tweezers for Sample; F: TEM Sampling Grid; G: Diffusion Flame 


\section{EXPERIMENTAL METHOD}

The study of high pressure generated soot reactions poses many significant challenges. From the construction and design of a high-pressure combustion vessel with a system capable of sampling at high pressures, to imaging in a microscope capable of providing an atmosphere and heat to the sample. The experiments were conducted in 4 major stages: high pressure flame soot synthesis, sampling and soot extraction, soot oxidation inside the ETEM, and image post processing.

Soot synthesis $\quad$ Soot synthesis was accomplished with a laminar co-flow non-premixed diffusion flame, with the details of the pressure vessel published in ${ }^{20}$. The ID of the fuel nozzle was a $4 \mathrm{~mm}$ non-tapered stainless-steel nozzle, and the burner system based on the nozzle and burner geometries found $\mathrm{in}^{21}$. The flame was fueled with ethylene gas diluted with nitrogen through the fuel nozzle, and dry lab air used in the co-flow to maintain flame stability. Fuel flow rates and dilution levels were chosen based on flame stability and soot loading at the high-pressure experimental conditions. Table 1 contains details of the fuel flow rates and coflow rates. Coflow rates were increased with pressure to maintain flame stability. By keeping hydrocarbon flow rates constant, increases in soot yield can be directly attributed to the increased pressures.

Table 1:Flow rates for each flame pressure setting

\begin{tabular}{|l|l|l|l|}
\hline Pressure (bar) & Ethylene $(\mathrm{mg} / \mathrm{s})$ & Nitrogen $(\mathrm{mg} / \mathrm{s})$ & Coflow $(\mathrm{g} / \mathrm{s})$ \\
\hline 1 & 0.8 & 2.14 & 0.64 \\
\hline 5 & 0.8 & 2.14 & 1.14 \\
\hline 10 & 0.8 & 2.14 & 1.64 \\
\hline
\end{tabular}




\begin{tabular}{|l|l|l|l|}
\hline 15 & 0.8 & 2.14 & 2.14 \\
\hline 20 & 0.8 & 2.14 & 2.64 \\
\hline 25 & 0.8 & 2.14 & 3.14 \\
\hline
\end{tabular}

Optical and physical access to the combustion chamber was provided through 4 ports separated by $90^{\circ}$ angles. Three of the flanges were used to provide optical access for the sampling cameras, and the ignition system while the fourth flange was fitted with the sampling setup. An ICCD camera located opposite of the sampling device was used to ensure sampling was done in the center of the flame. A high-speed camera was located at $90^{\circ}$ to the sampling device to record sampling. An Nd:YAG laser source (Ekspla model NL909-SH \#NLL252) at $1064 \mathrm{~nm}$ was used for ignition to maintain vessel pressure during ignition. The beam was expanded using a biconcave lens ( $f=-50$ $\mathrm{mm})$ and refocused with a plano-concave lens $(\mathrm{f}=125 \mathrm{~mm})$. Finally, to ignite the fuel the laser was focused at the burner using a plano-concave lens $(\mathrm{f}=400 \mathrm{~mm})$.

Soot Sampling The high-pressure sampling device was designed based on a system by Leschowski et. al. ${ }^{22}$ with some modifications, shown in Figure 2. The pneumatic piston was a Festo Standard cylinder model DSBC-63-300-F-PPVA-N3. A custom adaptor was designed to attach the pneumatic piston to the sealing housing. The sealing housing contains a $3.5 \mathrm{~cm}$ long Teflon tube with an ID of $23 \mathrm{~mm}$. Vacuum grease was used at the entrance of the tubing to ensure sealing. The grid holding adaptor was manufactured in house, with reverse acting tweezers mounted inside of the adaptor (Figure 2-3a). The sampling device was mounted to a flange that was secured to one of the vessels access ports. A quick release valve was used to secure the device 
to a ball valve. During sampling, vessel pressure could be maintained by opening or closing the ball valve while inserting or retrieving TEM grids from the tweezers.

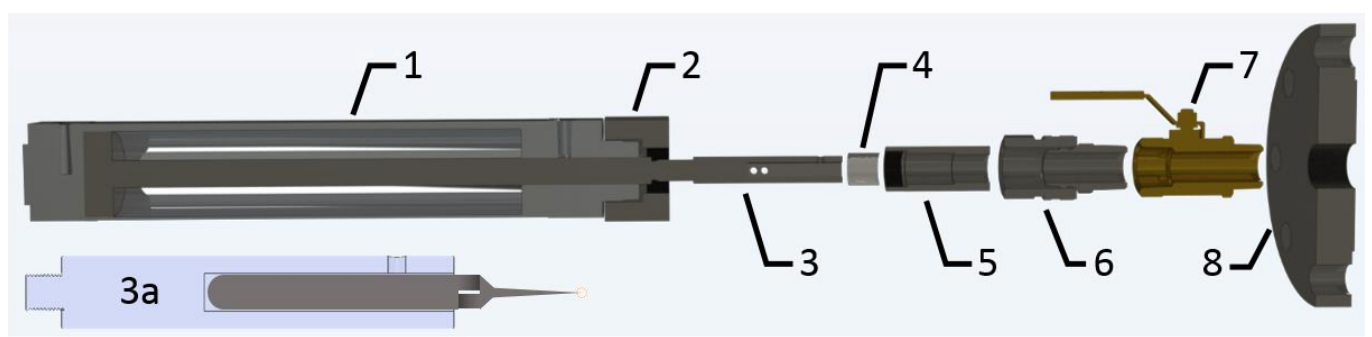

Figure 2: (1) pneumatic piston; (2) Adaptor to attach (1) to (5); (3) Tweezer holder adaptor; (4) Teflon seal; (5) Sealing chamber; (6) Swagelok quick release; (7) Ball valve; (8) Flange (Bottom Left Inset) (3a) Tweezer holder adaptor with reverse acting tweezers and TEM grid.

One challenge when using a thermophoretic sampling device is minimizing flame disturbances. In nonpremixed flames at high pressure, flame stability issues are exacerbated, and flame perturbations can originate from a variety of sources. During sampling, the rapid incursion of the pneumatic piston into the pressure vessel can be a source of such a disturbance. The pressure in the vessel is controlled with a back-pressure regulator (BPR). When the piston rapidly enters the vessel, this causes a minor increase in vessel pressure before the BPR can respond, and thus the incoming fuel is compressed, and the flame height momentarily decreases. To prevent flame perturbations caused by rapid pressure changes during sampling, the pneumatic piston was moved near the flame ( $\sim 1 \mathrm{~cm}$ away) using a Swagelok stainless steel low flow metering bypass valve, allowing the sample collection to occur before the flame was impacted by the pressure wave, shown by high speed imaging of the sampling setup in Figure 3. 

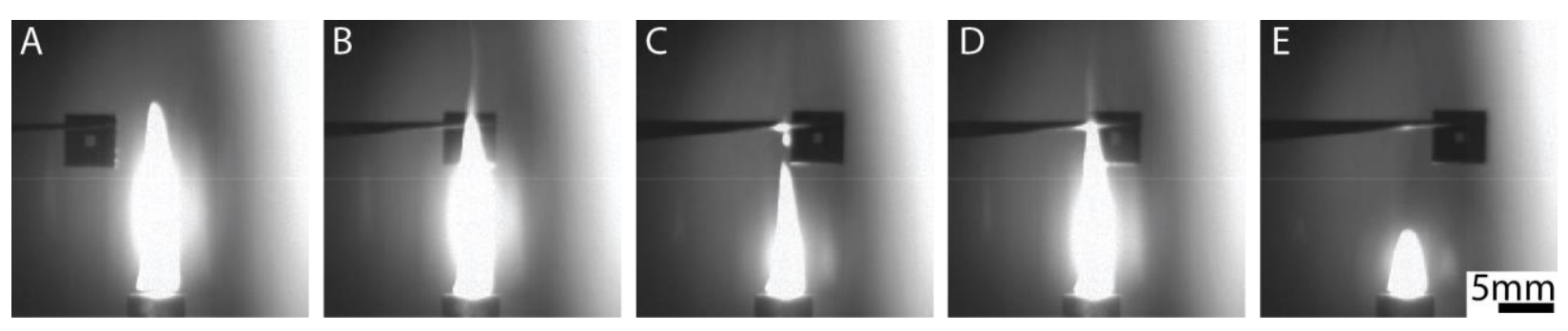

Figure 3: High speed sampling of soot from a high-pressure flame. Screenshots from: 15ATM_HS.AVI

Another cause of flame disturbance is from the TEM grid holder itself. To minimize flow perturbation during and following sampling, grids were held using Dumont N5 reverse acting tweezers. TEM grids were inserted into the flame vertically during sampling. Samples were taken at the same height in the centerline of the flame at the flame tip. Sampling was performed by slowly moving the grid to approximately $2.5 \mathrm{~cm}$ from the flame. The pneumatic piston was then triggered and the TEM grid moved into and past the flame zone. The flame was then extinguished, and the sample was retracted from the vessel and collected. The flame was then restarted using laser ignition for further sampling at high pressures. The tweezers were cleaned with acetone and dried between samplings to prevent contamination between different pressure conditions.

Oxidation Imaging To image the oxidation of the high-pressure soot, the ETEM technique relies on a customized transmission electron microscope (Hitachi HF3300) suitable for gas injection into the sample area, a microelectromechanical (MEMS) heating substrate where the sample is collected, and high sensitivity gas and temperature MEMS holder and control systems. While conventional transmission electron microscopes require a high vacuum through the entire column to allow imaging with electrons - the ETEM employs a series of additional pumps, apertures, and a custom gas injection sample holder to maintain a stable atmosphere surrounding the sample, while maintaining vacuum in all other parts of the microscope ${ }^{23,24}$. All these systems concurrently work together to allow imaging of soot oxidation at the nano scale. 
For the in situ studies, the soot samples are first collected thermophoretically from a flame onto 5x5mm Norcada in-Situ Microscopy MEMS chips (Figure 4-B). At their center, the MEMS chips have $50 \mathrm{~nm}$ thin windows surrounded by a heating element, seen in Figure 4-C. This design allows for sample imaging under heating up to $1100 \mathrm{~K}$. These chips are inserted into a custom Hitachi Gas/Heating holder (Figure 4-A), connected to the heating and gas systems, and inserted into the beam path of the microscope. The gas injection holder carries the reaction gas to the sample resulting in a quiescent gas cloud around the soot sample of $1 \mathrm{~Pa}$ - absolute. This low pressure compared to the pressures seen by soot in the diffusion flame will slow down the reaction ${ }^{25}$ from ms to minutes, and allow for real time observation and recording. Samples were collected for each video run on a chip, with a total of 10 in-situ chips collected.
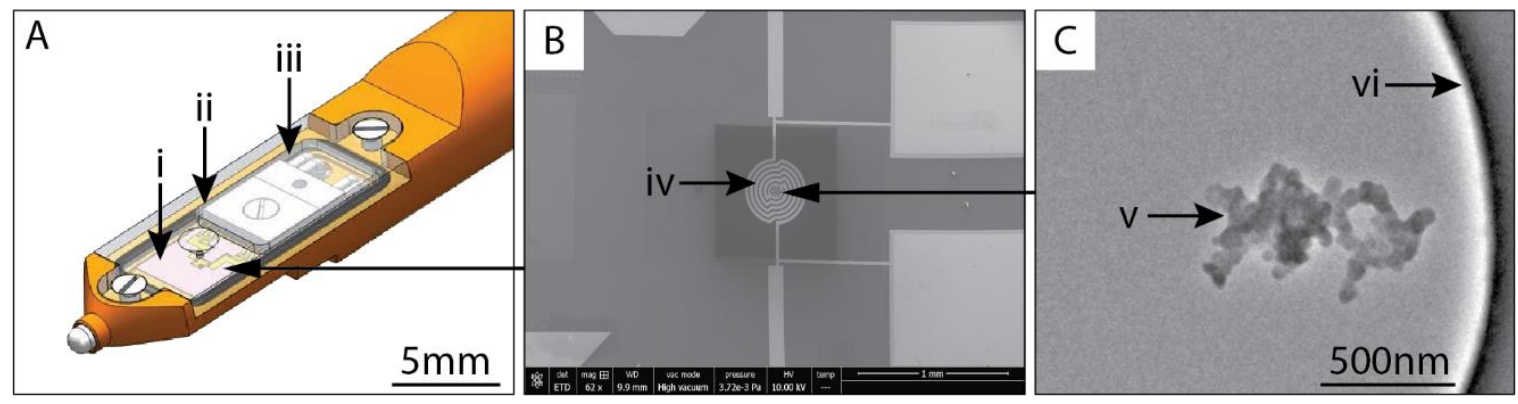

Figure 4A: ETEM Sample Chip Holder; i: Gas injection port; ii: Electrical Contacts; iii: MEMS sample chip.

Figure 4B: Image of the surface of the MEMS Sample Chip; iv: Spiral Heater surrounding the sample viewing area Figure 4C: Sample viewing window with sample; v: Soot sample; vi: heater edge

Post Processing Finally, the experimental videos are post processed with video and photo analysis tools such as ImageJ and Photoshop CC as shown in Figure 5. This post processing allows us to observe particle size changes, filter out background generated by the substrate, and more clearly track the reaction mechanism. The conventional post processing procedure is as follows: conversion to 24bit color, thresholding, background elimination, and color blending. The color blending applies a color enhancement to the particle, based on the luminance of the base image 
and the hue and saturation of the blend color. This preserves the grey levels in the image and is useful for coloring monochrome images and for tinting color images ${ }^{26}$.
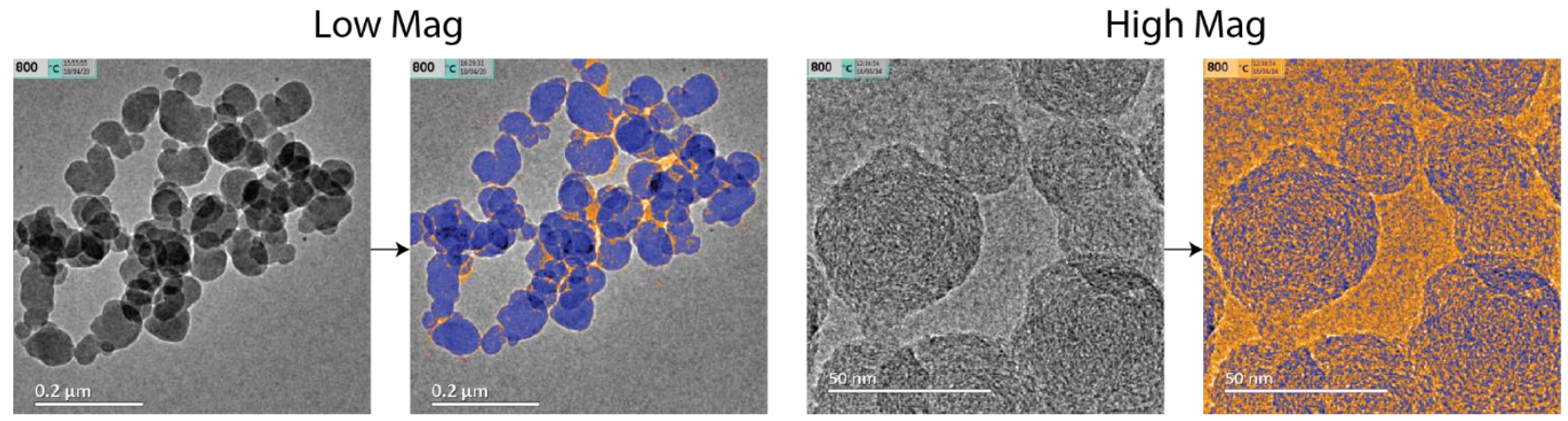

Figure 5: Before and After of video screenshots used for analysis

Cross sectional area changes during oxidation were also analyzed, in order to quantitively analyze the reactivity trends of the soot samples. While conventional modeling and experimental analysis measures primary particle diameter, this does not work on particles undergoing internal oxidation; where the outer shell of the particles remains mostly unchanged, while the core is hollowed out. For this reason, the cross sectional total area of the samples was measured using an automated ImageJ code which crops, thresholds, filters out background noise, and measures the total aggregate area. Using the same image brightness inside the microscope, and the same thresholding levels in the code, the aggregate area trends can be compared as a function of time by running the code on screenshots from the video. By plotting the change of area of each of the samples against the time in the video, a chart of relative reactivities can be generated for each set of magnifications and conditions. The automated processing code is included in the Appendix Figure 12.

\section{RESULTS}

We collected four soot samples with the combustor pressure set at each of the following settings: 1 bar, 5 bar, 10 bar, 15 bar, 20 bar, and 25 bar. As the combustor pressure increased, the observable 
flame diameter decreased. The flame height was relatively constant at $18.0 \pm 0.7 \mathrm{~mm}$ as seen in the two-color pyrometry measurements (Figure 6). The two-color pyrometry technique uses two color bands to measure the temperature of particles in the flame. As pyrometry can only measure temperatures on solid surfaces, this technique also highlights when solid soot particles begin to appear.

To maintain a constant mass flux and constant flame height, the flame density must increase. Higher density at elevated pressures promotes faster mixing and reaction rates, pushing the initial soot nucleation lower in the flame ${ }^{27}$. This can be seen in Figure 6, where the detection of soot occurs lower in the flame as pressures increase. This downward shift in soot nucleation and the decreasing temperature trends are consistent with experimental ${ }^{9,28}$ and modeling work ${ }^{29}$, showing earlier soot formation and a subsequent temperature drop due to radiative losses from the soot particles. This lower formation subsequently provides more residence time for the nucleated particles to grow, agglomerate, and graphitize. The extended residence time as pressures increase also restructures the soot nanoparticles, primarily made of amorphous non-ordered carbon, into larger soot aggregates with more defined internal structures ${ }^{6}$.

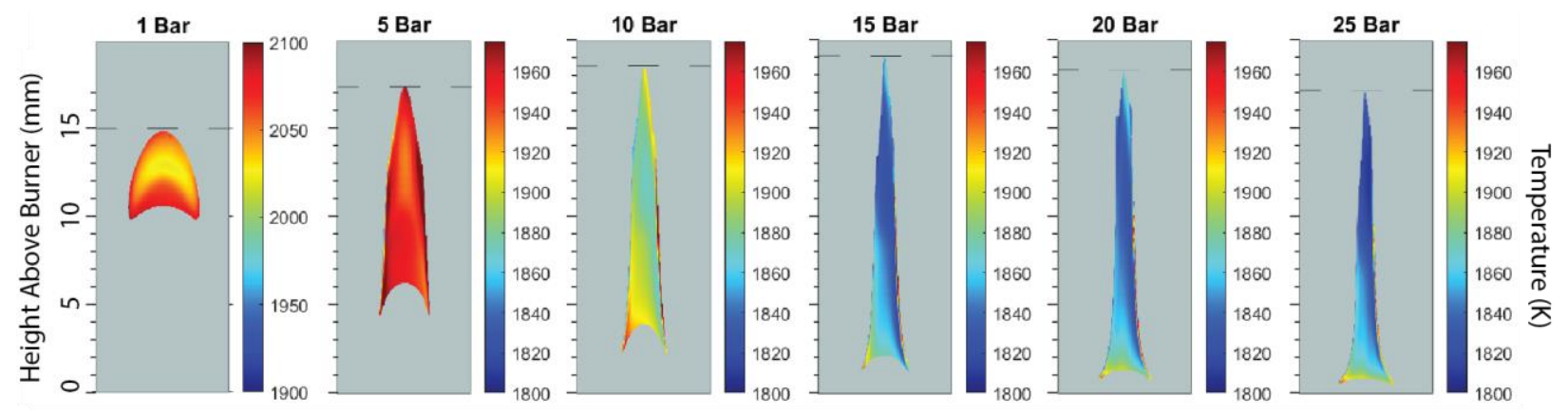

Figure 6: Temperature measurements as pressure increases measured with two color pyrometry 
Strzelec et al. found that changes in internal nanostructure can fundamentally alter the oxidation pathways and particle reactivity ${ }^{30}$. Longer soot residence times, from increasing the pressure from 5 bar to 25 bar, should generate larger, more graphitic particles which are more resistant to oxidation. To observe changes in particle structure, soot samples were extracted from the flame tip at $5,10,15,20$, and 25 bar-with the 1 bar case excluded because of its low-sooting behavior. After high-speed sampling, high-resolution transmission electron microscopy HRTEM provides a snapshot of the internal nanostructure of the soot aggregates as well as the average size of the particles. As pressures increase, the graphitic planes inside the soot particles not only grow but also rearrange into thicker and longer layers, parallel to the particle surface. This dramatic transition can be observed in Figure 7 as we move from 5 bar in image F to 25 bar in image J. These graphitic layers are highly unreactive with no available sites for oxygen to attach to and oxidize, protecting the particle from oxidation. Differences between this study and the study done by Commodo et. al. ${ }^{12}$ are primarily due to differences in fuel type, fuel dilution, and sampling pressures. In the Commodo study ${ }^{12}$, soot was sampled from a non-premixed laminar coflow flame running pure methane at 10,15 , and 20 bar. The soot appeared to be forming near the burner base at 10 bar based on soot luminosity images; thus, soot residence time does not change with pressure but only with sampling height. In the experiments performed for this work, temperature measurements show soot forming closer to the burner as pressure increases thus increasing soot residence time in the flame. Furthermore, methane and ethylene have been shown to form different soot nanostructures. HRTEM images of soot sampled from a McKenna burner found no changes in soot nanostructure in a premixed methane flame from young soot to mature soot whereas changes were observed in soot nanostructure with premixed ethylene ${ }^{31}$. 


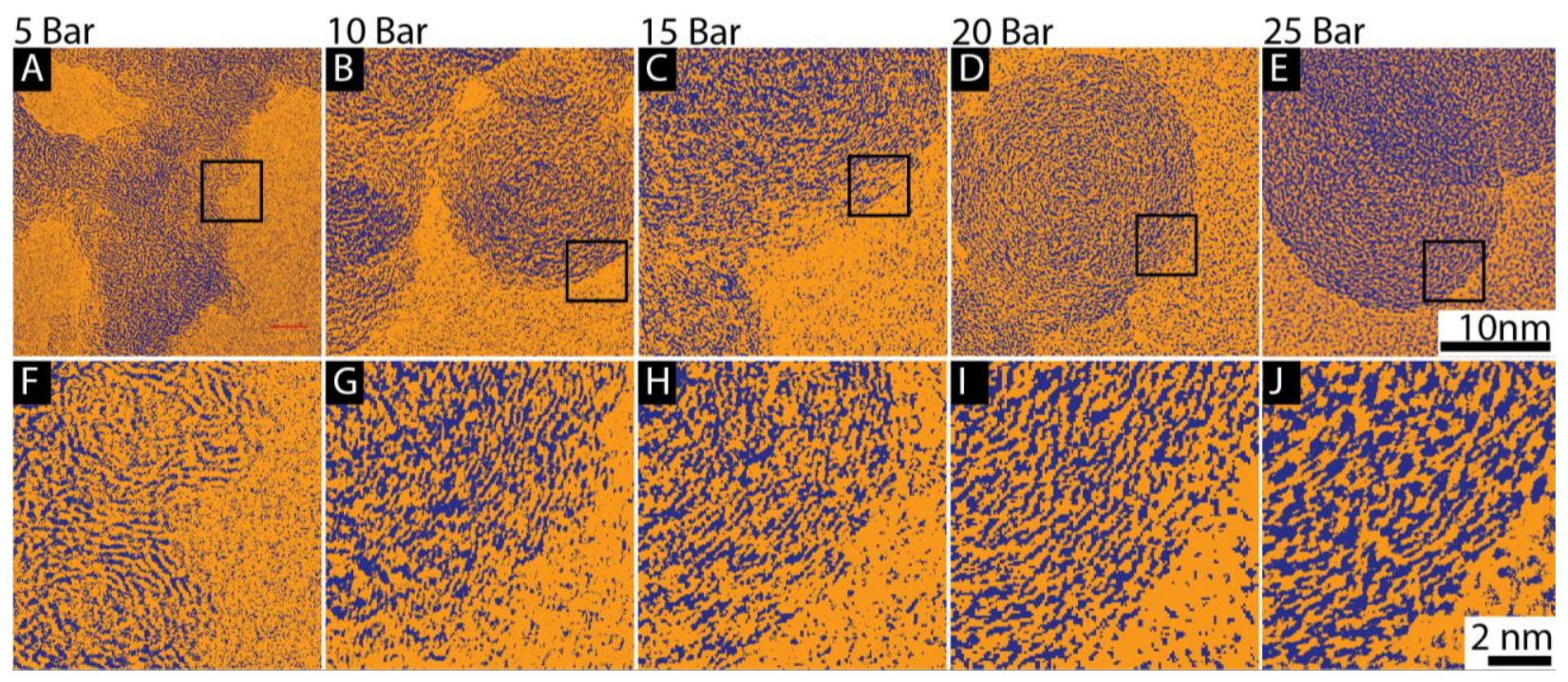

Figure 7: TEM and HRTEM Images of Soot Particles at High Pressures with carbon shown in purple and substrate in orange

To study how the increase in pressure and internal restructuring affects oxidation, in-situ oxidation tests were run inside the ETEM. For in-situ ETEM work, sample batches were collected from the flame tip thermophoretically onto Norcada $50 \mathrm{~nm}$ thin heating MEMS chips. Unlike conventional TEM grids, the MEMS chip allows for stable imaging of the samples at elevated temperatures inside the ETEM without thermal drift. The samples were brought up to $1100 \mathrm{~K}$ and oxidized in an ionized oxygen environment under the electron beam ${ }^{18}$. All tests were carried out under the same magnification, beam strength, and procedure, to ensure beam and ionization variance were minimized sample to sample.

Inside the ETEM, the conditions under which soot was generated were seen to have a significant impact on the oxidation behavior of the soot. The low-pressure soot seen in the 5 bar case (Figure 8) immediately burned aggressively from the inside. The post-processed images show nonoxidized soot in purple and oxidized soot in orange, with the time scales selected for each pressure to highlight the main oxidation steps for each sample. Oxygen diffused through openings in the thin graphitic shell, oxidizing the amorphous core and with only the graphitic shell remaining 2 
minutes into the test. The remaining shell burned away during the following hour. The highpressure 25 bar case proved extremely resilient, remaining mostly unaffected through 60 minutes of high temperature oxidation. The 15 bar case displayed both pathways; first with the outer shell oxidized, followed by internal oxidation as the surface oxidation formed openings through the shell. The videos for the 10 bar and 20 bar cases followed this same behavior, with significant internal burnup with the 10 bar samples and primarily surface oxidation in the 20 bar case.

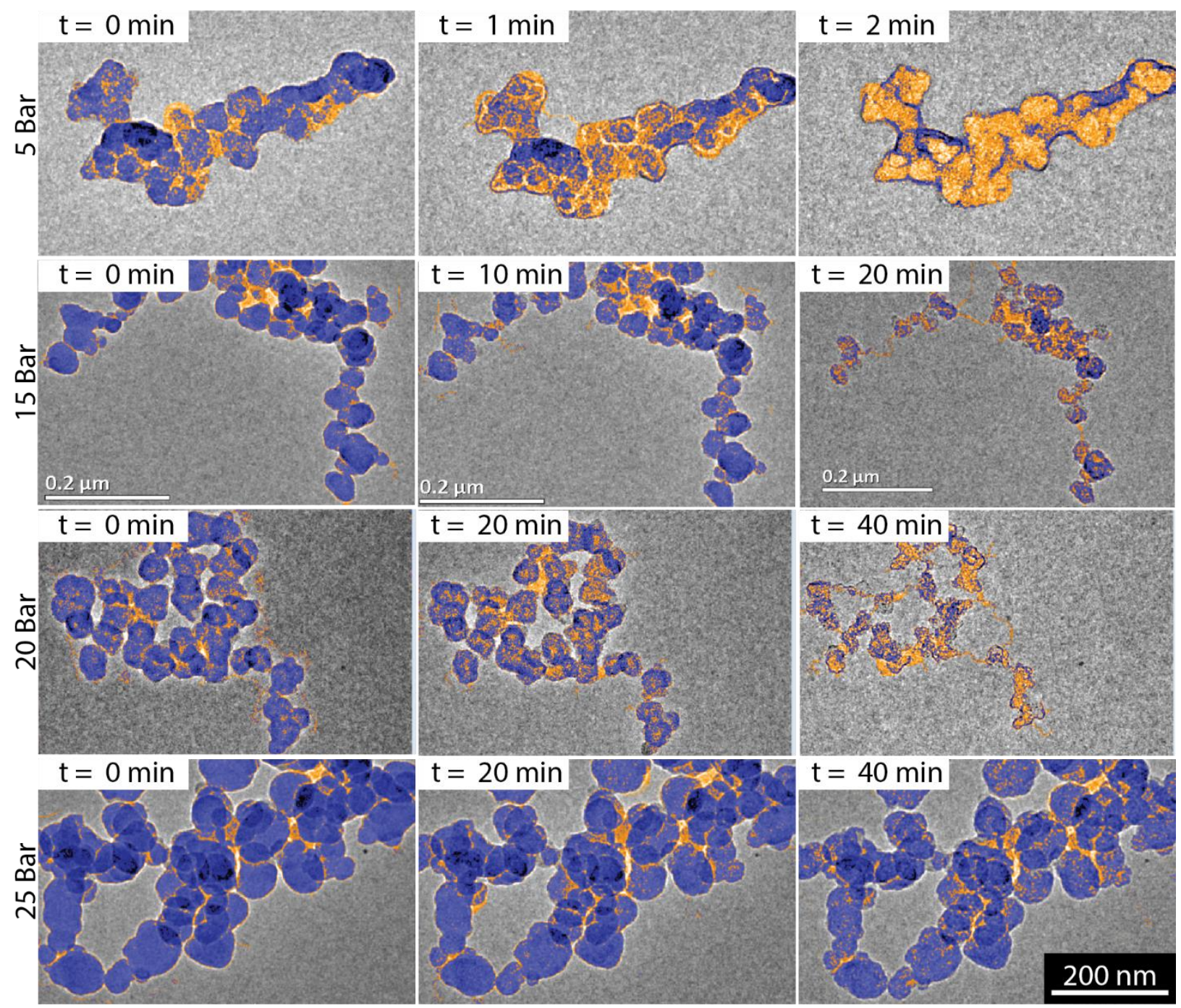


Figure 8: Screen captures of in-situ oxidation of soot. Purple-unburnt soot. Orange-substrate and oxygen diffusion areas. Time scales selected to reflect oxidation reaction rates. Screenshots from: 5ATM_LM.MP4, 15ATM_LM.MP4, 20ATM_LM.MP4 and 25ATM_LM.MP4

Screenshots were then taken of the videos and analyzed with an ImageJ code to determine the aggregate normalized cross-sectional area. This analysis allows for the comparison in sample reactivity trends regardless of the different oxidation pathways (internal, external, and in-between), and initial size. It can be seen in Figure 9 that as pressures increase, and the samples graphitize more and more, resisting oxygen diffusion, the reactivity of the soot samples decreases.
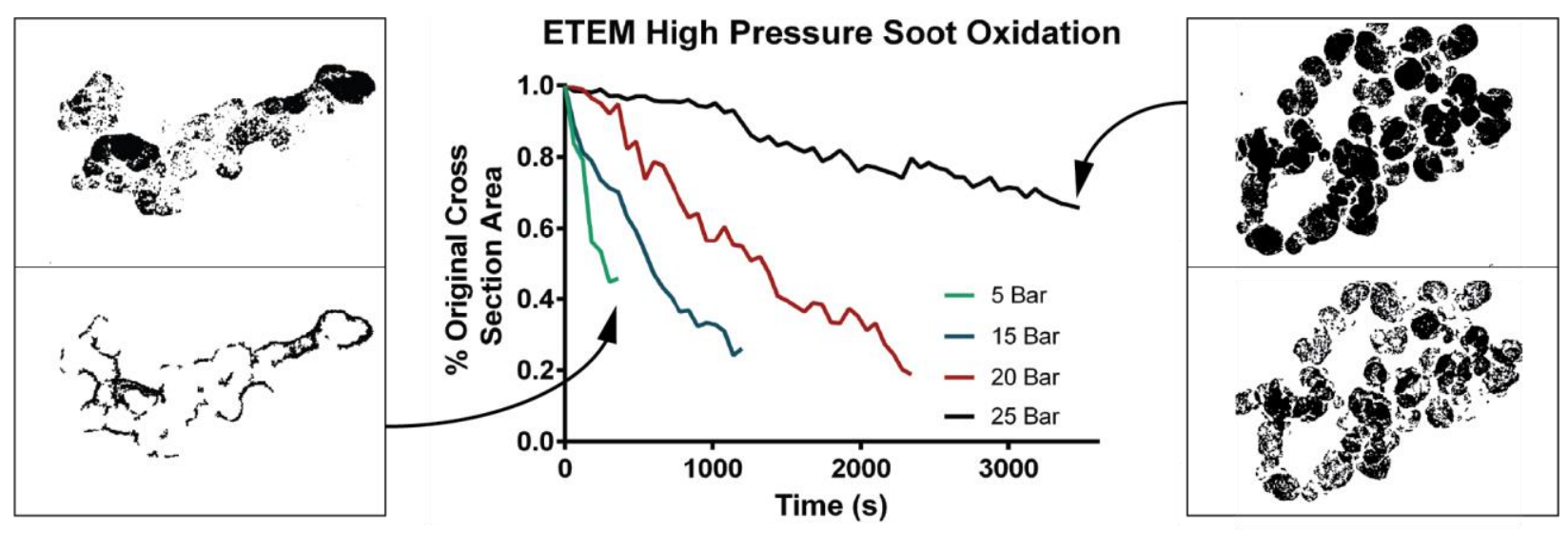

Figure 9: Cross sectional area change during oxidation analysis

To more clearly illustrate the oxygen diffusion and surface oxidation pathways, low and medium pressure soot samples were oxidized under a higher magnification in the ETEM, with a field of view limited to a few individual primary particles in the aggregate. In higher-magnification recordings in Figure 10-5 Bar, the 5 bar sample burns away from openings in the thin shell (a few of the shell openings are highlighted with arrows), while the remaining carbon sections rearrange into a shell over a time span of just over 10 minutes. This ordered shell is very unreactive but eventually burns away over an hour in the ETEM. Interestingly, as the amorphous carbon is oxidized, this opens active sites on the carbon surface, allowing for graphitization concurrently 
with oxidation. This phenomenon was first observed in 1966 by Heckman and Harling ${ }^{3}$, resulting in "Peanut Shell" like remnants - however, their ex-situ process failed to observe this transition, instead attributing it entirely to a previously graphitized structure.

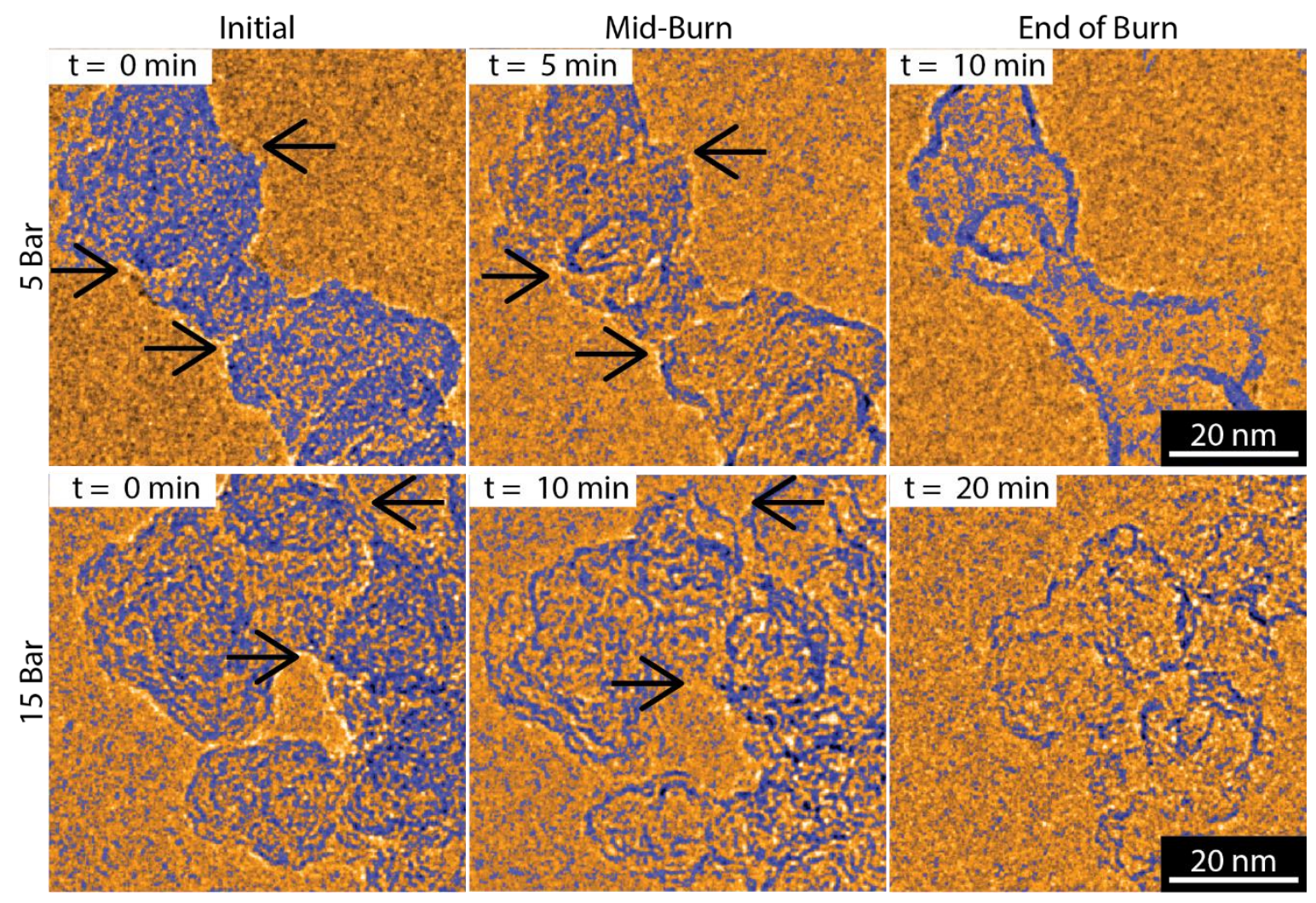

Figure 10: High magnification screen captures at $800^{\circ} \mathrm{C}$. Arrows highlight diffusion tunnels formed by oxidation. Screenshots from: 5ATM_HM.MP4, 15ATM_HM.MP4

\section{CONCLUSIONS}

Through the first real-time high-resolution visualization of the oxidation of high-pressure generated soot, the internal nanostructure changes and oxidation pathways of high-pressure soot were observed. While significant previous research asserts that increased pressure affects soot yields primarily through increased nucleation and lower flame temperatures, it was shown that these conditions are also favorable to the formation of a protective graphitic shell surrounding the 
soot particles. Due to the low reactivity of this graphitic shell, soot oxidation would first require the oxidation of the shell, opening tunnels for oxygen diffusion, followed by the internal oxidation of the amorphous carbon core. This pathway depends on the uniformity of the shell structure, as any flaws allow for the diffusion of oxygen into the particles and subsequently faster soot oxidation. These findings indicate that as combustor pressures increase in the hunt for higher efficiencies, more oxidation resilient soot is formed, requiring more aggressive filtration and mitigation strategies from combustion researchers. The change in oxidation pathways also demonstrates the need for more complex oxidation modeling - as current surface reaction models fail to capture the effects of internal nanostructure.

\section{ASSOCIATED CONTENT:}

All samples tested were recorded with high speed imaging during collection, and by the ETEM camera during oxidation. The file names are listed below.

Table 2: Experiment video data file names

\begin{tabular}{|l|l|l|l|}
\hline Pressure & Sample Collection Video & Oxidation Video 1 & Oxidation Video 2 \\
\hline 5 & 5ATM_HS & 5ATM_ETEM_LM & 5ATM_ETEM_HM \\
\hline 15 & 15ATM_HS & 15ATM_ETEM_LM & 15ATM_ETEM_HM \\
\hline 20 & 20ATM_HS & 20ATM_ETEM_LM & \\
\hline 25 & 25ATM_HS & 25ATM_ETEM_LM & \\
\hline
\end{tabular}


All unprocessed images are attached below for reference

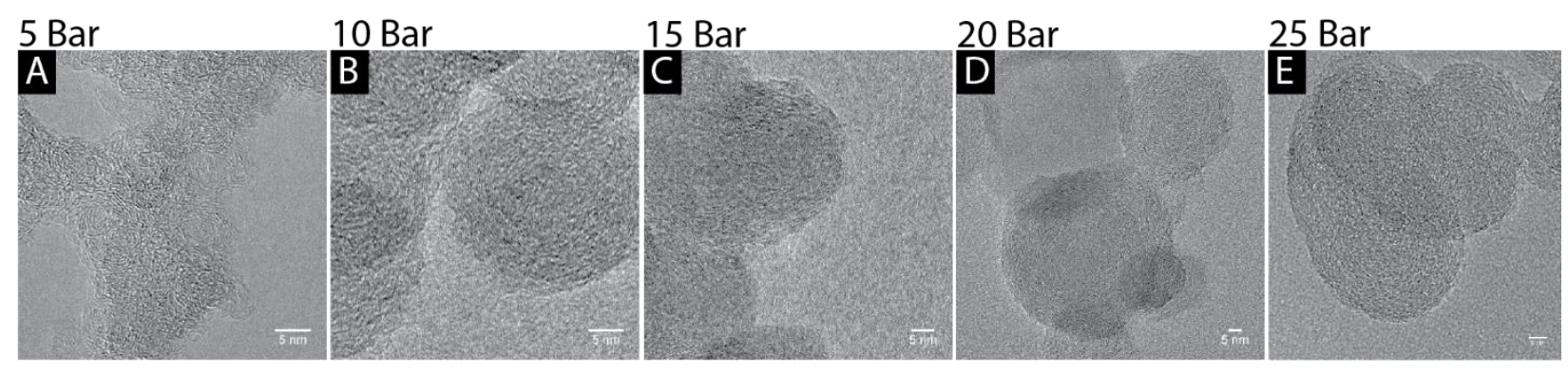

Figure 11: Original Figure 7 images with no editing or size normalization.

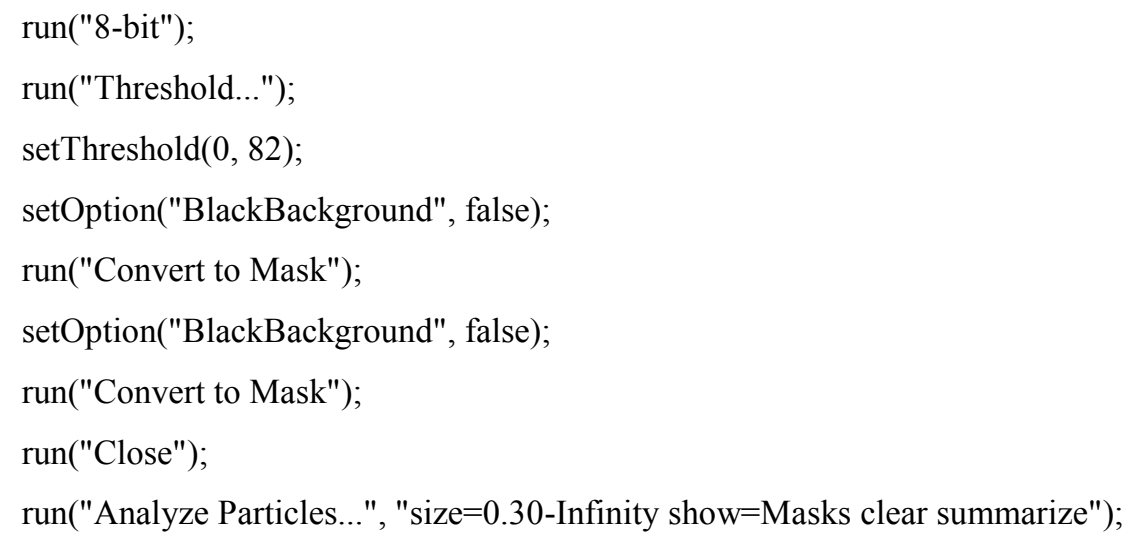

Figure 12: ImageJ Area Analysis Code for video Screenshots 

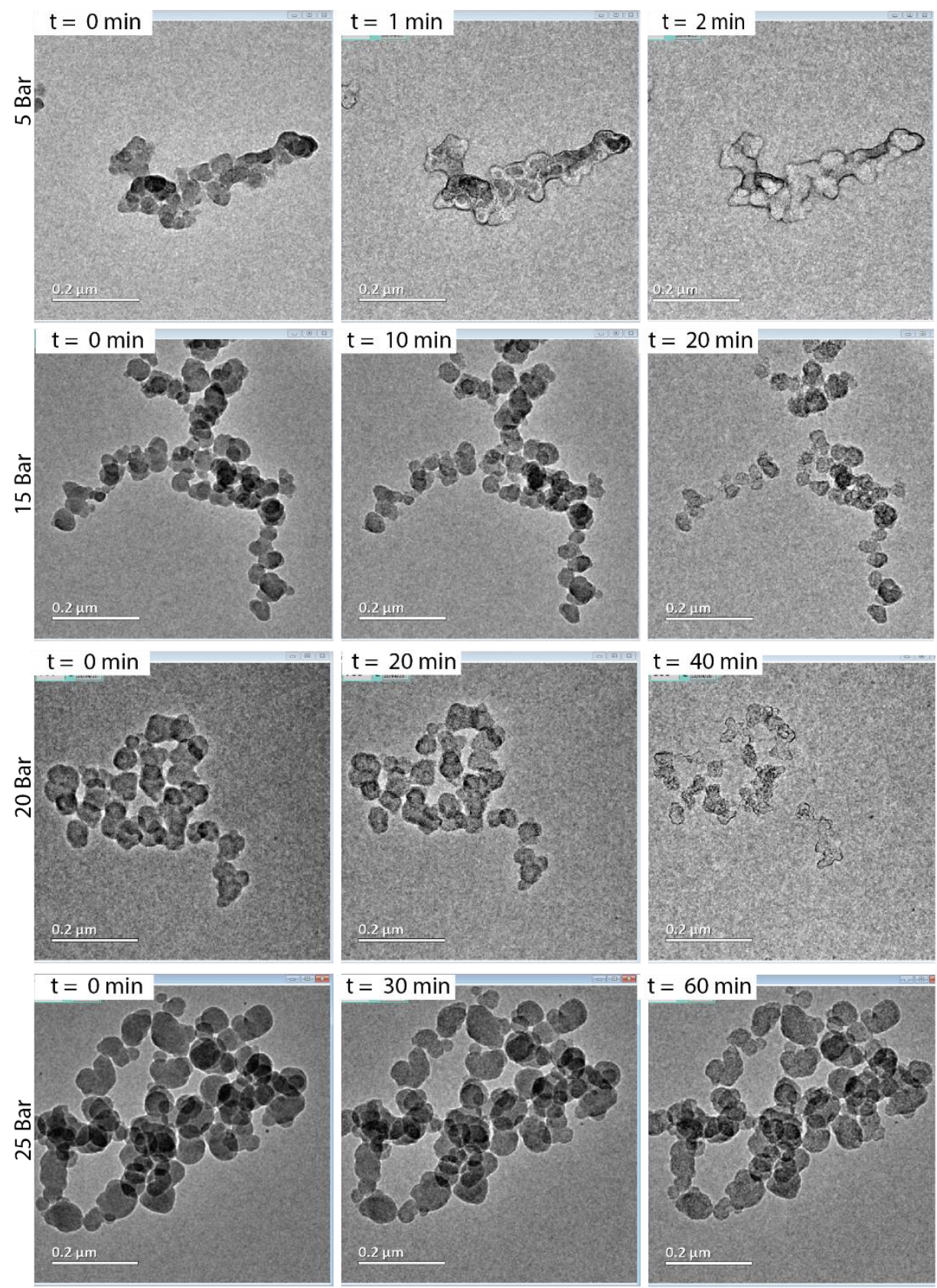

Figure 13: Unedited Figure 8 video screenshots 

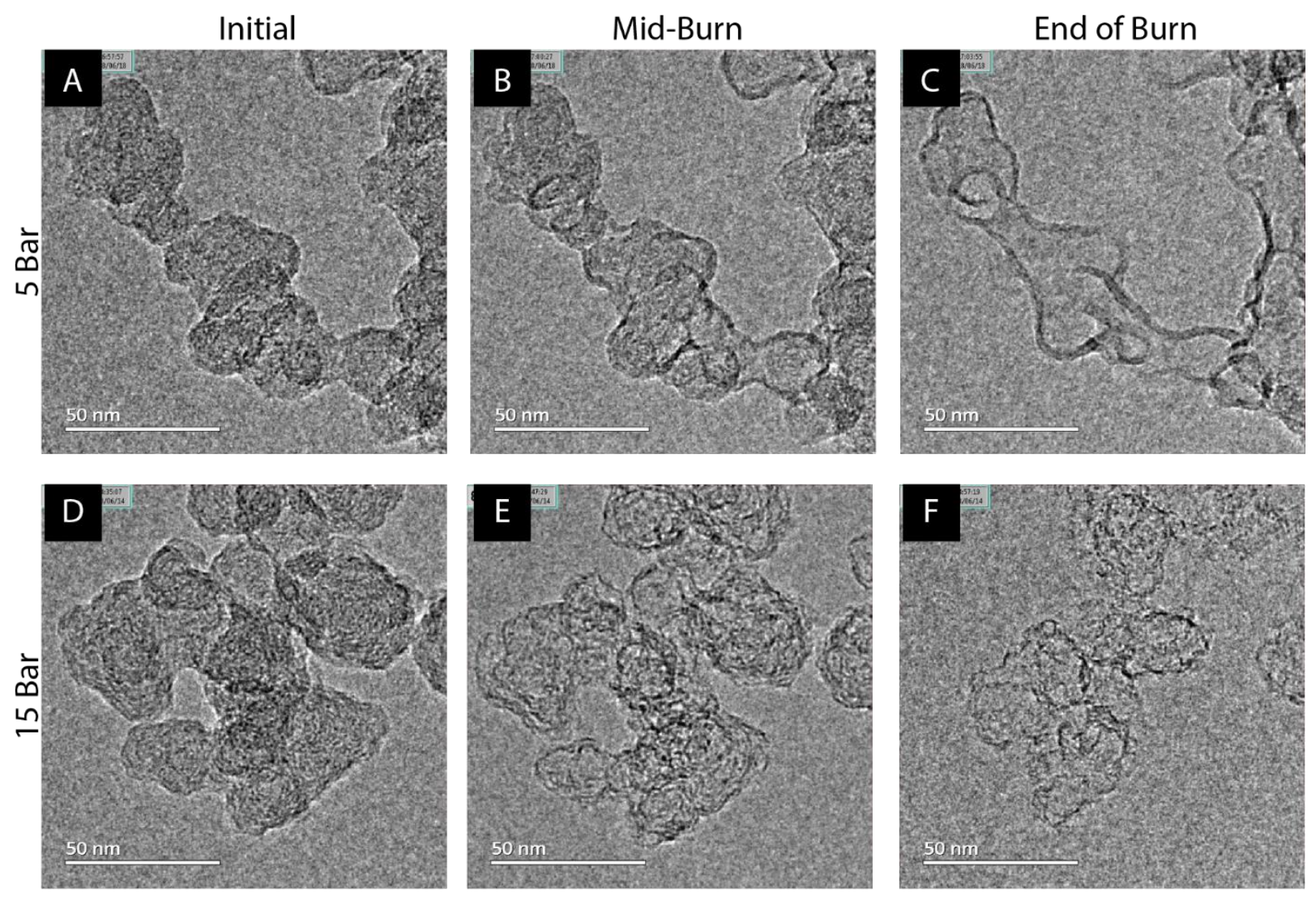

Figure 14: Unedited Figure 9 screenshots

\section{ACKNOWLEDGEMENTS:}

The authors would like to thank Hitachi Higher Technologies Canada and Norcada Inc. for their continued support with the development of this new technique. The authors would also like to acknowledge NSERC Canada and King Abdullah University of Science and Technology (KAUST) for project funding. We would also like to thank the OCCAM- UofT team, where all the microscopy was performed on the Hitachi HF3300. Finally, the authors would also like to thank Dr. Stas Dogel, Ali Naseri, and Dr. Pál Tóth for their invaluable technical expertise and discussions. 


\section{AUTHOR INFORMATION}

Dr. Murray J. Thomson

University of Toronto

Professor, Mechanical Engineering

Director, NSERC CREATE Program in Clean Combustion Engines

Laboratory: Combustion Research Laboratory (CRL) http://combustion.mie.utoronto.ca/

Email: murray.thomson@utoronto.ca | Tel: 416-573-1022 | Office: MC335

Website: http://www.mie.utoronto.ca/faculty/thomson.

\section{Notes}

The authors declare no competing financial interest. 


\section{REFERENCES}

1. IEA. Key World Energy Statistics 2016. Statistics (Ber). (2016). doi:10.1787/9789264039537-en

2. Ray, D. H. Particulate Matter. U.S. Environ. Prot. Agency (2004).

3. Heckman, F. A. \& Harling, D. F. Progressive Oxidation of Selected Particles of Carbon Black: Further Evidence for a New Microstructural Model. Rubber Chem. Technol. 39, 113 (1966).

4. Vander Wal, R. L. \& Tomasek, A. J. Soot oxidation: Dependence upon initial nanostructure. Combust. Flame (2003). doi:10.1016/S0010-2180(03)00084-1

5. Haynes, B. S. \& Wagner, H. G. Soot Formation. Prog. Energy Combust. Sci. (1981). doi:10.1016/0360-1285(81)90001-0

6. Franklin, R. E. Crystallite Growth in Graphitizing and Non-Graphitizing Carbons. Proc. $R$. Soc. A Math. Phys. Eng. Sci. (1951). doi:10.1098/rspa.1951.0197

7. Li, Z., Qiu, L., et al. The evolution of soot morphology and nanostructure in laminar diffusion flame of surrogate fuels for diesel. Fuel (2018). doi:10.1016/j.fuel.2017.09.036

8. Vander Wal, R. L. \& Tomasek, A. J. Soot nanostructure: Dependence upon synthesis conditions. Combust. Flame (2004). doi:10.1016/j.combustflame.2003.09.008

9. Flower, W. L. \& Bowman, C. T. Soot production in axisymmetric laminar diffusion flames at pressures from one to ten atmospheres. Symp. Combust. (1988). doi:10.1016/S00820784(88)80342-4 
10. McCrain, L. L. \& Roberts, W. L. Measurements of the soot volume field in laminar diffusion flames at elevated pressures. Combust. Flame (2005). doi:10.1016/j.combustflame.2004.10.005

11. kent, J. H. \& Wagner, H. G. Who do Diffusion flames Emit smoke. Combust. Sci. Technol. 41, 245-269 (1984).

12. Commodo, M., Joo, P. H., et al. Raman Spectroscopy of Soot Sampled in High-Pressure Diffusion Flames. Energy and Fuels (2017). doi:10.1021/acs.energyfuels.7b01674

13. Jo“, J., Appel, J., et al. Kinetic Modeling of Soot Formation with Detailed Chemistry and Physics: Laminar Premixed Flames of C 2 Hydrocarbons. (2000).

14. Bacsa, W. S., de Heer, W. A., et al. Raman spectroscopy of closed-shell carbon particles. Chem. Phys. Lett. (1993). doi:10.1016/0009-2614(93)87071-A

15. Yang, H., Li, X., et al. Experimental investigation into the oxidation reactivity and nanostructure of particulate matter from diesel engine fuelled with diesel/polyoxymethylene dimethyl ethers blends. Sci. Rep. 6, 37611 (2016).

16. Jaramillo, I. C., Gaddam, C. K., et al. Effect of nanostructure, oxidative pressure and extent of oxidation on model carbon reactivity. Combust. Flame (2015). doi:10.1016/j.combustflame.2014.12.006

17. Kholghy, M. R., Veshkini, A., et al. The core-shell internal nanostructure of soot - A criterion to model soot maturity. Carbon N. Y. (2016). doi:10.1016/j.carbon.2016.01.022

18. Koh, A. L., Gidcumb, E., et al. Oxidation of Carbon Nanotubes in an Ionizing Environment. 
Nano Lett. 16, 856-863 (2016).

19. Toth, P., Palotas, A. B., et al. The effect of oxidation pressure on the equilibrium nanostructure of soot particles. Combust. Flame (2015). doi:10.1016/j.combustflame.2015.02.009

20. Kailasanathan, A. \& Kumar, R. Experimental Investigations on Ethylene Laminar Flames at Elevated Pressures. (North Carolina State University, 2011).

21. Bennett, A., Amin, H. M. F., et al. Measurements of Pressure Effects on PAH Distribution and 2D Soot Volume Fraction Diagnostics in a Laminar Non-premixed Coflow Flame. Energy and Fuels (2018). doi:10.1021/acs.energyfuels.8b02179

22. Leschowski, M., Dreier, T., et al. An automated thermophoretic soot sampling device for laboratory-scale high-pressure flames. Rev. Sci. Instrum. (2014). doi:10.1063/1.4868970

23. Dogel, S., Hoyle, D., et al. Development of Environmental Specimen Holder for Hitachi S5500 UHR SEM Microscope. Microsc. Microanal. 17, 434-435 (2011).

24. Isakozawa, S., Nagaoki, I., et al. Design of a $300-\mathrm{kV}$ gas environmental transmission electron microscope equipped with a cold field emission gun. Reprod. Syst. Sex. Disord. 65, 353-362 (2016).

25. Neeft, T. X. Nijhuis, E. Smakman, M. Makkee, J. A. Moulijn, J. P. A. Kinetics of the oxidation of diesel soot. Fuel (1997). doi:10.1016/S0016-2361(97)00119-1

26. Adobe. Blending modes. Photoshop Help Manual (2017).

27. Karataş, A. E. \& Gülder, Ö. L. Soot formation in high pressure laminar diffusion flames. 
Progress in Energy and Combustion Science 38, 818-845 (2012).

28. Roberts, B. \& Fang, T. Soot Formation and Destruction in High- Pressure Flames with Real Fuels. (2011).

29. Eaves, N. A., Veshkini, A., et al. A numerical study of high pressure, laminar, sooting, ethane-air coflow diffusion flames. Combust. Flame 159, 3179-3190 (2012).

30. Strzelec, A., Wal, R. L. Vander, et al. NO2 Oxidation Reactivity and Burning Mode of Diesel Particulates. Top. Catal. 59, 686-694 (2016).

31. Alfè, M., Apicella, B., et al. Structure-property relationship in nanostructures of young and mature soot in premixed flames. Proc. Combust. Inst. (2009). doi:10.1016/j.proci.2008.06.193 